\title{
The sensitivity and significance of lateralized interictal slow activity on magnetoencephalography in focal epilepsy
}

\author{
Dario J. Englot, MD, $\mathrm{PhD}^{1,2,4}$, Srikantan S. Nagarajan, $\mathrm{PhD}^{1,4}$, Doris D. Wang, MD, $\mathrm{PhD}^{1,2,4}$, \\ John D. Rolston, MD, $\mathrm{PhD}^{1,2,4}$, Danielle Mizuiri, $\mathrm{BS}^{4}$, \\ Susanne M. Honma, RT ${ }^{4}$, Mary Mantle, REEG/EPT ${ }^{1,4}$, Phiroz E. Tarapore, M.D. ${ }^{1,2,4}$, \\ Robert C. Knowlton, MD ${ }^{1,3,4}$, Edward F. Chang, MD ${ }^{1,2,4}$, Heidi E. Kirsch MD ${ }^{1,3,4}$
}

${ }^{1}$ UCSF Comprehensive Epilepsy Center, ${ }^{2}$ Department of Neurological Surgery, ${ }^{3}$ Department of Neurology, ${ }^{4}$ Biomagnetic Imaging Lab, Department of Radiology and Biomedical Imaging, University of California, San Francisco, California, USA.

Correspondence to: Dario J. Englot, $\mathrm{MD}, \mathrm{PhD}$

Department of Neurological Surgery

University of California, San Francisco

505 Parnassus Avenue, Box 0112

San Francisco, California 94143-0112

Phone: (415) 353-3904 Email: dario.englot@ucsf.edu

Key words: epilepsy surgery, epileptogenic zone, MEG, magnetic source imaging, slowing Manuscript details: Words: 3,614. Text pages: 19. Tables: 3. Figures: 2. References: 31. Running title: MEG slowing in focal epilepsy Acknowledgements: This work was supported by the National Institutes of Health (F32NS086353 to DJE) and the following grants to SSN (NIH-R01DC010145, NIH-R01DC013979, NSF-BCS 1262297, DoD-W81XWH-13-1-0494). We thank all members of the UCSF Comprehensive Epilepsy Center and the Biomagnetic Imaging Lab for their support of this work, and for continued excellence in patient care. 


\section{Abstract}

Objective: Asymmetric large-amplitude slow activity is sometimes observed on interictal electroencephalography (EEG) in epilepsy. However, few studies have examined slowing during magnetoencephalography (MEG) recordings, which are performed primarily to localize interictal spikes. Also, no prior investigations have compared the sensitivity of MEG to scalp EEG in detecting slow rhythms.

Methods: We performed a retrospective cohort study of focal epilepsy patients who received MEG followed by surgical resection at our institution. We examined MEG, simultaneous EEG, and long-term EEG recordings for prominent asymmetric slow activity (delta-range, 1-4 Hz), and evaluated post-operative seizure outcomes.

Results: We studied 132 patients with $\geq 1$ year post-operative follow-up (mean, 3.6 years). Mean age was 27 (range, 3-68) years, and 55\% of patients were male. Asymmetric large-amplitude slow wave activity was observed on interictal MEG in 21 of $132(16 \%)$ patients. Interictal slowing lateralized to the hemisphere of resection in all but one $(95 \%)$ patient. Among the 21 patients with interictal MEG slowing, 11 (52\%) individuals had similarly lateralized EEG slowing, 7 patients had no EEG slowing, and 3 had bilateral symmetric EEG slowing.

Meanwhile, none of the 111 patients without lateralized MEG slowing had asymmetric EEG slowing, suggesting significantly higher sensitivity of MEG versus EEG in detecting asymmetric slowing $\left(\chi^{2}=63.4, p<0.001\right)$. MEG slowing was associated with shorter epilepsy duration with an odds ratio of 5.4 (1.7-17.0, 95\% confidence interval). At last follow-up, 92 (70\%) patients were seizure free (Engel I outcome), with no difference in seizure freedom rates between patients with $(71 \%)$ or without (69\%) asymmetric MEG slowing $\left(\chi^{2}=0.4, p=0.99\right)$. 
Significance: MEG has higher sensitivity than scalp EEG in detecting asymmetric slow activity in focal epilepsy, which reliably lateralizes to the epileptogenic hemisphere. Other uses of MEG beyond spike localization may further improve presurgical evaluations in epilepsy.

Key words: epilepsy surgery, epileptogenic zone, MEG, magnetic source imaging, slowing 


\section{Introduction}

Drug resistant focal epilepsy results in significant morbidity, but many patients can become free of seizures with surgical resection or ablation, although epilepsy surgery remains under-utilized (Cascino, 2008; Englot, 2015; Englot et al., 2012). Successful epilepsy surgery depends critically on accurate delineation of the epileptogenic zone (EZ), resulting in seizure freedom in approximately two-thirds of patients with temporal lobe epilepsy (TLE) and one-half of individuals with extra-temporal lobe epilepsy (ETLE) (Englot et al., 2013; Englot et al., 2014; Spencer and Huh, 2008). Electroencephalography (EEG) and magnetoencephalography (MEG) are valuable clinical tools for localizing interictal spike activity related to the EZ, but other abnormal rhythms are also often observed. Previous authors have described large-amplitude monomorphic slow wave activity (delta-range, 1-4 Hz) on interictal scalp EEG in focal epilepsy patients, which may lateralize to the hemisphere harboring the EZ (Blume et al., 1993; Koutroumanidis et al., 2004; Smith, 2005). Furthermore, a few investigations have reported lateralized slow activity during interictal MEG recordings (Baayen et al., 2003; Ishibashi et al., 2002; Kamada et al., 1998). However, it is not well understood how scalp EEG and MEG compare in the detection and localization of interictal slow rhythms.

Compared to scalp EEG, MEG possesses higher spatio-temporal resolution without signal distortions from the skull and scalp (Kirsch et al., 2007; Zumer et al., 2007), and is a valuable noninvasive diagnostic modality in the presurgical evaluation of epilepsy patients (Kharkar and Knowlton, 2014). In focal epilepsy patients, we recently examined the role of MEG-based studies in EZ localization and prediction of post-operative seizure outcome, by analyzing interictal spike localization (Englot et al., 2015b) and regional functional connectivity 
alterations (Englot et al., 2015a). However, the sensitivity of interictal slow activity on MEG has not been previously studied in detail or compared to that of scalp EEG. Also, it is unknown whether asymmetric MEG slowing reliably lateralizes to the side of the EZ.

In the present study, we examine asymmetric interictal slow activity among MEG recordings in 132 presurgical epilepsy patients. We compare the laterality and location of interictal slow activity on MEG with that observed during simultaneous EEG recordings and long-term EEG monitoring, to assess the sensitivity of MEG versus EEG for asymmetric slowing. We also explore potential relationships between MEG slowing and other variables of interest, including duration of disease and interictal spike localization. Finally, we evaluate the concordance between asymmetric interictal slowing on MEG and the laterality of surgical resection, and examine seizure outcomes $\geq 1$ year after surgery.

\section{Methods}

\subsection{Patients}

Retrospectively we identified MEG recordings from 310 epilepsy patients referred for studies at the Biomagnetic Imaging Laboratory (BIL) at the University of California, San Francisco (UCSF) between June 1, 2004 and June 30, 2013. Out of these, we examined 144 patients who received surgical resection for focal epilepsy at our center after MEG recordings. We excluded 12 patients without at least one year of follow-up after surgery, and analyzed 132 patients. In general, $43 \%$ of individuals who receive extra-temporal resection and $36 \%$ of patients who receive temporal lobe resection for drug-resistant epilepsy at our institution during the study period received MEG. Referral criteria are not strict in our practice, but MEG is typically 
performed in cases in which surgery is being considered, but uncertainty remains regarding epileptogenic zone localization. Prior to MEG, patients have high-resolution 3T magnetic resonance imaging (MRI) with specific epilepsy protocols, interictal scalp EEG, formal neuropsychological evaluation, and long-term video monitoring as an inpatient with scalp EEG (with the exception of two patients in the present study who did not have long-term EEG). After these diagnostics, if the epileptogenic zone is not clearly delineated, or if there is a lack of concordance between electrophysiology, neuroimaging, and clinical symptomatology, MEG is ordered. For individuals who received multiple MEG recordings before surgery, only the last session prior to resection was considered. Of note, the patient population in the present study of interictal slow waves was previously studied in our recent investigation of interictal spike localization in epilepsy (Englot et al., 2015b). Procedures were all in compliance with UCSF clinical research policies, and research protocols were approved by the UCSF Committee on Human Research.

\subsection{MEG data acquisition and analysis}

Simultaneous MEG and EEG recordings were collected inside a magnetically shielded room, with a CTF whole-head 275 channel axial gradiometer system (VSM MedTech, Port Coquitlam, British Columbia). MEG recordings were collected from each patient in a passband of 0-75 $\mathrm{Hz}$ (300 Hz sample rate). Twenty-one channel scalp EEG recordings were collected simultaneously using a modified international 10-20 system including subtemporal electrodes. Thirty to forty minutes of spontaneous data were obtained in intervals of 10-15 min with the patient asleep and awake. The position of the patient's head in the dewar relative to the MEG sensors was 
determined using indicator coils before and after each recording interval to verify adequate sampling of the entire field. The data were then bandpass filtered offline at $1-70 \mathrm{~Hz}$. Other details of our simultaneous MEG and EEG recording methods have been previously described (Kaiboriboon et al., 2010).

Slow wave activity and spike activity on MEG were visually identified by a certified EEG technologist (MM) and were then confirmed by a board-certified clinical neurophysiologist and epileptologist (HEK). Large-amplitude slow (delta-range, 1-4 Hz) wave activity was noted on both MEG and simultaneous EEG recordings, including the laterality and location of the activity. Only patients with slow activity deemed to be very clearly lateralized or focal by the interpreting neurophysiologist were designated as having asymmetric slowing for the purposes of the present study. Furthermore, long-term EEG recordings in the same patients were examined, and the presence and location of large-amplitude slow activity was recorded, in addition to standard clinical evaluation of interictal spike activity and ictal activity. One goal of our study is to determine the rate at which asymmetric large-amplitude slow activity is recognized on MEG or scalp EEG by traditional human interpretation of abnormal electrographic activity, in the same way that seizures or interictal spikes are recognized. Therefore, we did not apply a strict electrographic cut-off for this activity. However, the amplitude of the asymmetric slow activity seen on MEG was approximately $1 \mathrm{pT}$.

We employed the criteria defined by the International Federation of Clinical Neurophysiology (IFCN) for the detection of interictal spike activity on simultaneous MEG/EEG recordings (Deuschl and Eisen, 1999). MEG spikes were chosen for analysis based on field map, duration ( $<80 \mathrm{~ms}$ ), lack of associated artifact, and morphology. Spike onset, defined as the rising deflection of the first sharp negativity from the baseline, was marked and equivalent current 
dipoles were fit using commercial software provided by CTF Systems (VSM MedTech, Port Coquitlam, British Columbia). Sources with a goodness of fit greater than $90 \%$ were accepted. Co-registration of dipoles to MRI scans was employed using fiducials (preauricular points and nasion) to create magnetic source images (MSI) of dipoles superimposed on the anatomic MRI. The authors examined these results and classified the dipoles according to location. The concordance between MEG spike localization with the EZ was examined at the lobar and sublobar level and contrasted with the area of resection in all patients. If the area harboring spike dipoles encompassed more than one lobar region of epileptogenic tissue, but the area of resection also overlapped these multiple regions (e.g., right lateral fronto-temporal spikes preceding a fronto-temporal cortical resection), spikes were considered concordant. Spike activity was considered concordant and specific if no appreciable (>10\%) dipoles were observed in other lobes. In the setting of another distal region with $>10 \%$ dipoles, spike activity was considered non-specific (a 10\% limit was chosen to allow occasional spurious spikes that do not co-localize with the majority of dipoles during a recording session). Other cases included those in which dipoles were ipsilateral but in a different area compared to the reference region (concordant lateralization only), and instances of discordant lateralization with $>50 \%$ of dipoles being contralateral. Examination and localization of slow wave and spike activity were performed while blinded to the patient's seizure outcome.

\subsection{Evaluation of clinical data and seizure outcomes}

We retrospectively reviewed outpatient and inpatient provider notes, diagnostic and laboratory reports, operative reports, and pathology records for all patients. We recorded patient gender, 
age, handedness, surgical history, epilepsy duration, medication history, and side of surgery.

Details regarding patients' epilepsy history and seizure semiology were obtained from pre- and post-operative assessments by epileptologists. Operative decisions were made by a comprehensive team of epileptologists, neurosurgeons, neuroradiologists, neuropsychologists, as well as other practitioners. Pre-operative evaluation included MEG, structural MRI, and scalp EEG, and often also included positron emission tomography (PET), neuropsychological evaluation, and long-term video-EEG monitoring with or without extra-operative electrocorticography (ECoG) including subdural and/or depth electrodes. Intra-operative interictal ECoG was also performed in the majority of operations. Surgeries were customized to incorporate epileptogenic regions and cerebral lesions while preserving eloquent cortex, where applicable. For patients with mesial TLE, an anterior temporal lobectomy was performed, including tailored resection of the lateral temporal cortex, amygdala, and hippocampus. Resections involved multiple lobes in cases with presumed multi-focal or hemispheric epilepsy syndromes. All specimens were analyzed by neuropathologists. Seizure outcome was classified by the last patient follow-up with the epileptologist using a modified Engel classification system (Engel et al., 1993).

\subsection{Statistical analysis}

To identify potential associations between factors of interest and the presence or absence of asymmetrical slowing on MEG, univariate analysis was performed using a $\chi^{2}$ test for categorical variables (e.g. gender) or an unpaired Student's $t$-test for continuous variables (e.g. age). Only variables showing a $p$ value $<0.20$ on univariate analysis were then entered into a multivariate 
logistic regression model in a backwards fashion. Thus, the multivariate model was built to identify variables significantly associated with asymmetric slow activity and potential interactions between these variables. Odds ratios (ORs) were calculated with a $95 \%$ confidence

interval (CI). Separate $\chi^{2}$ tests were also used to examine the relationship of MEG slowing to the presence or absence of asymmetric slowing on EEG, and the presence or absence of postoperative seizure freedom (Engel class I outcome) across all patients. Statistical significance was assessed at $p<0.05$ for all tests, with statistical analyses performed using SPSS version 22 (IBM, Somers, NY).

\section{Results}

\subsection{Patient characteristics}

We evaluated 132 patients with drug-resistant focal epilepsy who received MEG for localization of the EZ, followed by resective epilepsy surgery at our institution. All individuals had at least 1 year of post-operative follow-up (mean, 3.6 years). Seventy-three (55\%) individuals were male, and mean age $( \pm$ SEM) at the time of surgery was 27.3 years (range, 3-68 years). Other patient demographics are summarized in Table 1.

\subsection{Characteristics of slow wave activity}

Asymmetric monomorphic large-amplitude slow (1-4 Hz) wave activity was observed on interictal MEG recordings in 21 of $132(16 \%)$ patients, including 14 males and seven females. Figure 1 depicts example MEG recordings in a 33-year-old male with drug-resistant right TLE 
and a normal MRI, in which MEG revealed large-amplitude slowing in the right temporal lobe that was not seen on simultaneous EEG recordings. As summarized in Table 2, among the 21 patients with asymmetric MEG slowing, slow activity appeared diffusely hemispheric in 11 $(52 \%)$ individuals, but was localized to a single lobar region in $8(38 \%)$ patients, including seven patients with TLE. Both frontal and temporal lobe slowing was noted in two individuals. Interictal spike activity was also observed in $17(81 \%)$ of these 21 patients, lateralizing to the same hemisphere as the slow activity in $16(94 \%)$ of those cases. Overall, interictal MEG slow activity lateralized to the same hemisphere as the surgical site in all but one (95\%) patient, suggesting that slowing strongly predicts EZ laterality.

\subsection{Sensitivity of MEG and EEG for slowing}

To compare the sensitivity of MEG versus EEG in the detection of slow wave activity, examination of interictal EEGs was performed in all 132 patients. This analysis included shortterm simultaneous EEG recordings performed during the MEG session, and long-term EEG recordings from the epilepsy monitoring unit. Exception was made for two patients who did not undergo long-term scalp EEG monitoring, one of whom did have MEG slowing (patient no. 20 in Table 2), and in these patients only simultaneous EEG/MEG recordings were examined. As summarized in Figure 2, interictal scalp EEG detected lateralized slowing in only $11(52 \%)$ of the 21 patients with MEG slowing. Among the 10 patients with interictal MEG slowing who did not have asymmetric slowing on EEG, 3 individuals had bilateral but symmetric EEG slow activity, while 7 patients has no prominent slow activity present on EEG. Furthermore, MEG detected lateralized slowing in all $(100 \%)$ cases of slow activity seen on EEG, as none of the 111 
patients without asymmetric MEG slowing had asymmetric slow activity detected on EEG. Over all, this difference suggests a significantly higher sensitivity of MEG compared to EEG for the detection of asymmetric slowing $\left(\chi^{2}=63.4, p<0.001\right)$. Interestingly, of the 21 patients with asymmetric MEG slow activity, 9 individuals underwent long-term ECoG monitoring with intracranial electrodes. The large-amplitude slow activity was observed on ECoG on all 9 of these patients, regardless of whether asymmetric slowing was seen ( 5 patients) or was not seen (4 patients) on scalp EEG.

\subsection{Factors associated with slowing}

Both univariate and multivariate analyses were used to examine various factors for potential association with asymmetric MEG slowing, as summarized in Table 3. On univariate analysis, patients with MEG slowing were significantly younger than those without slowing, although no other differences in demographics were noted (Table 3A). In examining epilepsy characteristics, patients with a shorter duration of epilepsy and those with an extra-temporal EZ were more likely to harbor lateralized MEG slowing (Table 3B), although no difference in pre-operative diagnostic findings were noted between patients with or without slowing (Table 3C). In particular, interictal spike activity on MEG localized specifically to the EZ in $52 \%$ of patients, was non-specific in $27 \%$ of individuals, and was not seen in $22 \%$ of patients, but the rates of MEG slow activity (14-18\%) did not differ significantly among these groups (Table 3C). On multivariate analysis, shorter epilepsy duration was significantly and independently predictive of the presence of asymmetric MEG slowing $(p=0.01)$. Specifically, compared to individuals with epilepsy duration $\geq 10$ years, epilepsy duration $<10$ years was associated with an increased 
likelihood of MEG slowing with an odds ratio of $5.4(1.7-17.0,95 \% \mathrm{CI})$. Other variables were not significantly associated with MEG slowing on multivariate analysis.

\subsection{Post-operative seizure outcomes}

At last post-operative follow-up, 92 (70\%) patients were free of disabling seizures (Engel IA-D), including 67 (51\%) individuals who achieved complete seizure freedom (Engel IA). Other outcomes were Engel II, Engel III, or Engel IV in 13 (10\%), 17 (13\%), and 10 (8\%) individuals, respectively. There was no difference in seizure freedom (Engel I) rates between patients with $(71 \%)$ or without $(69 \%)$ asymmetric MEG slowing $\left(\chi^{2}=0.4, p=0.99\right)$.

\section{Discussion}

\subsection{Summary of slow wave activity}

Among 132 patients who underwent interictal MEG in the present study, lateralized largeamplitude slow wave activity was observed in $21(16 \%)$ patients. Interictal slowing lateralized to the hemisphere of planned resection in all but one (95\%) of these individuals. Among the 21 patients with interictal MEG slowing, similarly lateralized EEG slowing was present in only 11 (52\%) of individuals, while none of the 111 patients without asymmetric MEG slowing had asymmetric EEG slowing. This suggests significantly higher sensitivity of MEG versus EEG in detecting asymmetric slowing, which is likely related to improved spatio-temporal resolution with MEG without signal distortions from the skull and scalp that limits signal propagation with EEG.(Zumer et al., 2007) Shorter epilepsy duration was associated with an increased likelihood 
of MEG slowing, which has not been previously reported, to our knowledge. We did not observe a relationship between the presence of interictal slowing and post-operative seizure outcome.

\subsection{Previous MEG and EEG studies of lateralized slowing}

Although this is the first study to directly compare the ability of MEG and EEG to detect asymmetric slow activity in focal epilepsy, other groups have previously observed MEG slowing in this patient population. Ishibashi and colleagues examined focal slow wave activity on MEG in 17 patients with MTLE, and found that this rhythm was typically most prominent over the temporal area, always lateralized to the side of resection (Ishibashi et al., 2002). Baaven et al. examined MEG recordings in 20 patients with brain tumor-associated epilepsy, and observed interictal slowing in $65 \%$ of individuals, most often in the region of the lesion (Baayen et al., 2003). Finally, Kamada and colleagues described MEG slowing in the sensorimotor areas of three children with rolandic epilepsy (Kamada et al., 1998). These previous studies suggest that MEG may be a useful tool for the detection of asymmetric interictal slowing, and our present results support this conclusion.

On EEG, generalized large-amplitude slow wave activity is a frequent finding in encephalopathy of various causes (Ponten et al., 2013). However, asymmetric or focal slowing is more often noted interictally in EEG studies of focal epilepsy (Smith, 2005). For instance, Blume and colleagues observed lateralized slow activity during interictal EEG on the side of the EZ in 46 TLE patients with this abnormal rhythm (Blume et al., 1993). Koutroumanidis et al., described similar slow wave activity that was often lateralized to the EZ in surgical TLE patients, although in most patients slowing provided little additional localizing value beyond other 
diagnostic findings (Koutroumanidis et al., 2004). Interestingly, concordance between EEG spiking and slowing was a significant predictor of a favorable post-operative outcome. In our patient population, MEG spike localization did predict seizure freedom after surgery, but the presence of slowing alone did not, and only one patient in our study had discordant lateralization between spike and slow activity. Given that asymmetric slowing observed with MEG was also noted on ECoG in patients with intracranial recordings, it is likely that the increased sensitivity of MEG (over scalp EEG) to slow activity does not likely reflect an inherent quality of MEG recordings, but rather represents a limitation of scalp EEG, in which electrographic signals are degraded by the scalp and skull.

\subsection{Functional neuroimaging correlates of interictal slow activity}

Functional neuroimaging correlates of interictal slow waves in epilepsy have also been investigated in a small number of studies. Manganotti et al. examined simultaneous functional MRI (fMRI) and EEG recordings in eight patients with focal epilepsy, and observed significant activation of the blood-oxygen level dependent (BOLD) signal that co-localized with interictal slowing (Manganotti et al., 2008). Laufs and colleagues reported a similar finding of BOLD signal activation in the right frontal lobe of a single patient, co-localizing with the region harboring increased delta oscillations presumed to represent the epileptogenic zone (Laufs et al., 2006). It is not clear, however, why increased BOLD signal rather than BOLD decreases would be seen a region of interictal slowing that presumably represents the EZ. During interictal PET studies, the EZ typically displays diminished metabolism, and focal hypometabolism on PET has been previously reported in regions of interictal slowing (Koutroumanidis et al., 1998). However, 
it is important to recognize that cerebral metabolism and fMRI signals are only indirectly related (Englot et al., 2009). Further investigation utilizing various functional neuroimaging modalities in patients with focal interictal slowing will be necessary to address this issue.

\subsection{Ictal versus interictal slow activity}

While the present study examined interictal slow activity in epilepsy, it is important to distinguish this phenomenon from ictal slow activity that occurs during seizures. In particular, while interictal slow activity appears to co-localize with the EZ or epileptogenic hemisphere, ictal neocortical slow activity during partial seizures likely represents depressed cortical activity distal from the EZ. Previous intracranial EEG investigations have noted widespread frontoparietal neocortical slow activity during temporal lobe seizures that is associated with ictal loss of consciousness (Blumenfeld et al., 2004b; Englot et al., 2010). While seizure activity results in regional increases in cerebral blood flow, this ictal neocortical slow activity produces widespread decreases in blow flow (Blumenfeld et al., 2004a). Ictal neocortical slowing has also been studied in rodent models, and found to be associated with decreases in BOLD signal, cerebral blood flow, cerebral blood volume, and cortical metabolism, while the spread of fast seizure activity causes increases in each of these parameters (Englot et al., 2008; Englot et al., 2009). The mechanism of ictal slowing appears to be related to aberration of normal activity in subcortical structures important for arousal, as previously described in the "network inhibition hypothesis"(Danielson et al., 2011; Englot and Blumenfeld, 2009; Englot et al., 2009). Thus, asymmetric interictal slowing in focal epilepsy, which may aid in localization of the EZ between 
seizures, should not be confused with ictal cortical slow activity, which likely represents inhibition of distal networks during seizure activity.

\subsection{Multimodal use of MEG in the presurgical epilepsy evaluation}

While MEG may be useful for the identification of interictal slowing in epilepsy with higher sensitivity than EEG, only a minority of patients in the present study had significant lateralized slowing on visual inspection. Therefore, a multimodal approach to MEG for EZ localization is needed. As we have previously reported in this same patient population, dipole fitting of interictal spikes is successful in approximately $75 \%$ of focal epilepsy patients, and these dipoles localize specifically to the EZ in about two-thirds of those individuals (Englot et al., 2015b). We also recently described that functional connectivity analysis using MEG may have a role in the preoperative epilepsy evaluation, as patients with regional increases in connectivity at the planned resection site are most likely to achieve post-operative seizure freedom (Englot et al., 2015a). The investigation of high frequency oscillations (HFOs) may also be of interest in MEG, as the localizing value of these signals has become increasingly appreciated in epilepsy (Bragin et al., 2010). Finally, beyond localization, MEG is a valuable tool for non-invasive mapping of eloquent cortex including language, somatosensory, motor, and visual function in patients with epilepsy or other neurosurgical disorders (Findlay et al., 2012; Tovar-Spinoza et al., 2008). By combing various complementary analysis paradigms in future studies, the effective use of MEG in the presurgical epilepsy evaluation may be better optimized.

\subsection{Study limitations}


There are several limitations to the present study. Most obviously, prominent lateralized interictal slow wave activity was observed in a minority of focal epilepsy patients, so caution should be taken in generalizing our findings. Also, selection bias is likely present in our study, given that patients with an EZ that is difficult to localize are more likely to undergo MEG at our center. Next, asymmetric slowing on both MEG and EEG was interpreted using visual analysis by a certified neurophysiologist, and thus may be prone to bias. However, we utilized visual analysis to increase the applicability to our findings to other practices, as the vast majority of MEG and EEG studies in clinical practice are interpreted strictly by visual neurophysiological examination. Nevertheless, advanced adaptive spatial filtering techniques can be utilized for automated spike localization, and this technology may be investigated for slow wave detection in future studies.

\subsection{Conclusions}

Prominent lateralized interictal slow wave activity is seen in some patients with focal epilepsy, and MEG appears to have a higher sensitivity for this rhythm than scalp EEG. In nearly all patients with interictal slow activity, slowing is observed the side of the presumed EZ, and may aid presurgical lateralization in patients without focal interictal spikes. In this study, lateralized slow activity was observed more commonly in patients with a shorter duration of epilepsy. Other uses of MEG beyond spike localization may further improve presurgical evaluation in epilepsy. 


\section{Acknowledgements/disclosures:}

The authors have no conflicts of interest to disclose. This work was supported by the National Institutes of Health (F32-NS086353 to DJE) and the following grants to SSN (NIHR01DC010145, NIH-R01DC013979, NSF-BCS 1262297, DoD-W81XWH-13-1-0494). We thank all members of the UCSF Comprehensive Epilepsy Center and the Biomagnetic Imaging Lab for their support of this work, and for continued excellence in patient care. 


\section{References}

Baayen, J.C., de Jongh, A., Stam, C.J., de Munck, J.C., Jonkman, J.J., Trenite, D.G., Berendse, H.W., van Walsum, A.M., Heimans, J.J., Puligheddu, M., Castelijns, J.A., Vandertop, W.P., 2003. Localization of slow wave activity in patients with tumor-associated epilepsy. Brain topography 16, 85-93.

Blume, W.T., Borghesi, J.L., Lemieux, J.F., 1993. Interictal indices of temporal seizure origin. Annals of neurology 34, 703-709.

Blumenfeld, H., McNally, K.A., Vanderhill, S.D., Paige, A.L., Chung, R., Davis, K., Norden, A.D., Stokking, R., Studholme, C., Novotny, E.J., Zubal, I.G., Spencer, S.S., 2004a. Positive and negative network correlations in temporal lobe epilepsy. Cereb Cortex 14, 892-902.

Blumenfeld, H., Rivera, M., McNally, K.A., Davis, K., Spencer, D.D., Spencer, S.S., $2004 b$. Ictal neocortical slowing in temporal lobe epilepsy. Neurology 63, 1015-1021.

Bragin, A., Engel, J., Jr., Staba, R.J., 2010. High-frequency oscillations in epileptic brain. Curr Opin Neurol 23, 151-156.

Cascino, G.D., 2008. When drugs and surgery don't work. Epilepsia 49 Suppl 9, 79-84.

Danielson, N.B., Guo, J.N., Blumenfeld, H., 2011. The default mode network and altered consciousness in epilepsy. Behavioural neurology 24, 55-65.

Deuschl, G., Eisen, A., 1999. Recommendations for the practice of clinical neurophysiology: guidelines of the International Federation of Clinical Neurophysiology. Electroencephalogr Clin Neurophysiol Suppl 52, 1-304. 
Engel, J., Van Ness, P., Rasmussen, T., Ojemann, L., 1993. Outcome with respect to epileptic seizures, in: Engel, J. (Ed.), Surgical Treatment of the Epilepsies, ed 2. Raven Press, New York, pp. 609-621.

Englot, D.J., 2015. The persistent under-utilization of epilepsy surgery. Epilepsy research 118, 68-69.

Englot, D.J., Blumenfeld, H., 2009. Consciousness and epilepsy: why are complex-partial seizures complex? Progress in brain research 177, 147-170.

Englot, D.J., Hinkley, L.B., Kort, N.S., Imber, B.S., Mizuiri, D., Honma, S.M., Findlay, A.M., Garrett, C., Cheung, P.L., Mantle, M., Tarapore, P.E., Knowlton, R.C., Chang, E.F., Kirsch, H.E., Nagarajan, S.S., 2015a. Global and regional functional connectivity maps of neural oscillations in focal epilepsy. Brain : a journal of neurology 138, 2249-2262.

Englot, D.J., Lee, A.T., Tsai, C., Halabi, C., Barbaro, N.M., Auguste, K.I., Garcia, P.A., Chang, E.F., 2013. Seizure types and frequency in patients who "fail" temporal lobectomy for intractable epilepsy. Neurosurgery 73, 838-844.

Englot, D.J., Mishra, A.M., Mansuripur, P.K., Herman, P., Hyder, F., Blumenfeld, H., 2008. Remote effects of focal hippocampal seizures on the rat neocortex. The Journal of neuroscience : the official journal of the Society for Neuroscience 28, 9066-9081.

Englot, D.J., Modi, B., Mishra, A.M., DeSalvo, M., Hyder, F., Blumenfeld, H., 2009. Cortical deactivation induced by subcortical network dysfunction in limbic seizures. The Journal of neuroscience : the official journal of the Society for Neuroscience 29, 13006-13018.

Englot, D.J., Nagarajan, S.S., Imber, B.S., Raygor, K.P., Honma, S.M., Mizuiri, D., Mantle, M., Knowlton, R.C., Kirsch, H.E., Chang, E.F., 2015b. Epileptogenic zone localization using 
magnetoencephalography predicts seizure freedom in epilepsy surgery. Epilepsia 56, 949-958.

Englot, D.J., Ouyang, D., Garcia, P.A., Barbaro, N.M., Chang, E.F., 2012. Epilepsy surgery trends in the United States, 1990-2008. Neurology 78, 1200-1206.

Englot, D.J., Raygor, K.P., Molinaro, A.M., Garcia, P.A., Knowlton, R.C., Auguste, K.I., Chang, E.F., 2014. Factors associated with failed focal neocortical epilepsy surgery. Neurosurgery 75, 648-656.

Englot, D.J., Yang, L., Hamid, H., Danielson, N., Bai, X., Marfeo, A., Yu, L., Gordon, A., Purcaro, M.J., Motelow, J.E., Agarwal, R., Ellens, D.J., Golomb, J.D., Shamy, M.C., Zhang, H., Carlson, C., Doyle, W., Devinsky, O., Vives, K., Spencer, D.D., Spencer, S.S., Schevon, C., Zaveri, H.P., Blumenfeld, H., 2010. Impaired consciousness in temporal lobe seizures: role of cortical slow activity. Brain : a journal of neurology 133 , 3764-3777.

Findlay, A.M., Ambrose, J.B., Cahn-Weiner, D.A., Houde, J.F., Honma, S., Hinkley, L.B., Berger, M.S., Nagarajan, S.S., Kirsch, H.E., 2012. Dynamics of hemispheric dominance for language assessed by magnetoencephalographic imaging. Annals of neurology 71, 668-686.

Ishibashi, H., Simos, P.G., Castillo, E.M., Maggio, W.W., Wheless, J.W., Kim, H.L., Venkataraman, V., Sanders, D.K., Breier, J.I., Zhang, W., Davis, R.N., Papanicolaou, A.C., 2002. Detection and significance of focal, interictal, slow-wave activity visualized by magnetoencephalography for localization of a primary epileptogenic region. Journal of neurosurgery $96,724-730$. 
Kaiboriboon, K., Nagarajan, S., Mantle, M., Kirsch, H.E., 2010. Interictal MEG/MSI in intractable mesial temporal lobe epilepsy: spike yield and characterization. Clinical neurophysiology : official journal of the International Federation of Clinical Neurophysiology 121, 325-331.

Kamada, K., Moller, M., Saguer, M., Kassubek, J., Kaltenhauser, M., Kober, H., Uberall, M., Lauffer, H., Wenzel, D., Vieth, J., 1998. Localization analysis of neuronal activities in benign rolandic epilepsy using magnetoencephalography. Journal of the neurological sciences $154,164-172$.

Kharkar, S., Knowlton, R., 2014. Magnetoencephalography in the presurgical evaluation of epilepsy. Epilepsy \& behavior : E\&B 46, 19-26.

Kirsch, H.E., Mantle, M., Nagarajan, S.S., 2007. Concordance between routine interictal magnetoencephalography and simultaneous scalp electroencephalography in a sample of patients with epilepsy. Journal of clinical neurophysiology : official publication of the American Electroencephalographic Society 24, 215-231.

Koutroumanidis, M., Binnie, C.D., Elwes, R.D., Polkey, C.E., Seed, P., Alarcon, G., Cox, T., Barrington, S., Marsden, P., Maisey, M.N., Panayiotopoulos, C.P., 1998. Interictal regional slow activity in temporal lobe epilepsy correlates with lateral temporal hypometabolism as imaged with 18FDG PET: neurophysiological and metabolic implications. Journal of neurology, neurosurgery, and psychiatry 65, 170-176.

Koutroumanidis, M., Martin-Miguel, C., Hennessy, M.J., Akanuma, N., Valentin, A., Alarcon, G., Jarosz, J.M., Polkey, C.E., 2004. Interictal temporal delta activity in temporal lobe epilepsy: correlations with pathology and outcome. Epilepsia 45, 1351-1367. 
Laufs, H., Hamandi, K., Walker, M.C., Scott, C., Smith, S., Duncan, J.S., Lemieux, L., 2006. EEG-fMRI mapping of asymmetrical delta activity in a patient with refractory epilepsy is concordant with the epileptogenic region determined by intracranial EEG. Magnetic resonance imaging 24, 367-371.

Manganotti, P., Formaggio, E., Gasparini, A., Cerini, R., Bongiovanni, L.G., Storti, S.F., Mucelli, R.P., Fiaschi, A., Avesani, M., 2008. Continuous EEG-fMRI in patients with partial epilepsy and focal interictal slow-wave discharges on EEG. Magnetic resonance imaging 26, 1089-1100.

Ponten, S.C., Tewarie, P., Slooter, A.J., Stam, C.J., van Dellen, E., 2013. Neural network modeling of EEG patterns in encephalopathy. Journal of clinical neurophysiology : official publication of the American Electroencephalographic Society 30, 545-552.

Smith, S.J., 2005. EEG in the diagnosis, classification, and management of patients with epilepsy. Journal of neurology, neurosurgery, and psychiatry 76 Suppl 2, ii2-7.

Spencer, S., Huh, L., 2008. Outcomes of epilepsy surgery in adults and children. The Lancet. Neurology 7, 525-537.

Tovar-Spinoza, Z.S., Ochi, A., Rutka, J.T., Go, C., Otsubo, H., 2008. The role of magnetoencephalography in epilepsy surgery. Neurosurgical focus 25, E16.

Zumer, J.M., Attias, H.T., Sekihara, K., Nagarajan, S.S., 2007. A probabilistic algorithm integrating source localization and noise suppression for MEG and EEG data. NeuroImage 37, 102-115. 


\section{Figure Legends}

\section{Figure 1: Example simultaneous MEG/EEG recordings in a patient with asymmetric MEG} slowing. Recordings were performed in a 33-year-old male with a seven-year history of drug resistant epilepsy and a normal MRI. MEG revealed large-amplitude slow (delta) activity in the right temporal lobe that was not appreciated on simultaneous EEG recordings (shown on the right), or on long-term scalp EEG recordings (not shown). Enlarged 1-s epochs showing a few temporal lobe channels that do not (bottom left) or do (bottom right) exhibit slow activity are also provided. No interictal spikes were captured during the MEG session. After a long-term intracranial monitoring study, the patient underwent tailored right temporal lobe resection, and remained seizure free (Engel class I outcome) four years after surgery.

Figure 2: Increased sensitivity of MEG for asymmetric slowing compared to EEG. Among 132 patients, asymmetric slowing was identified on MEG recordings in 21 (15.9\%) individuals. Among these 21 patients with MEG slowing, asymmetric EEG slowing was observed in 11 $(52.3 \%)$ individuals, while $10(47.7 \%)$ patients had either no EEG slowing or bilateral EEG slowing. Zero (0\%) of the 111 patients without MEG slowing showed asymmetric slowing on EEG. This results in a significantly higher sensitivity of MEG than EEG for asymmetric slowing $\left(\chi^{2}=63.4, p<0.001\right)$ 


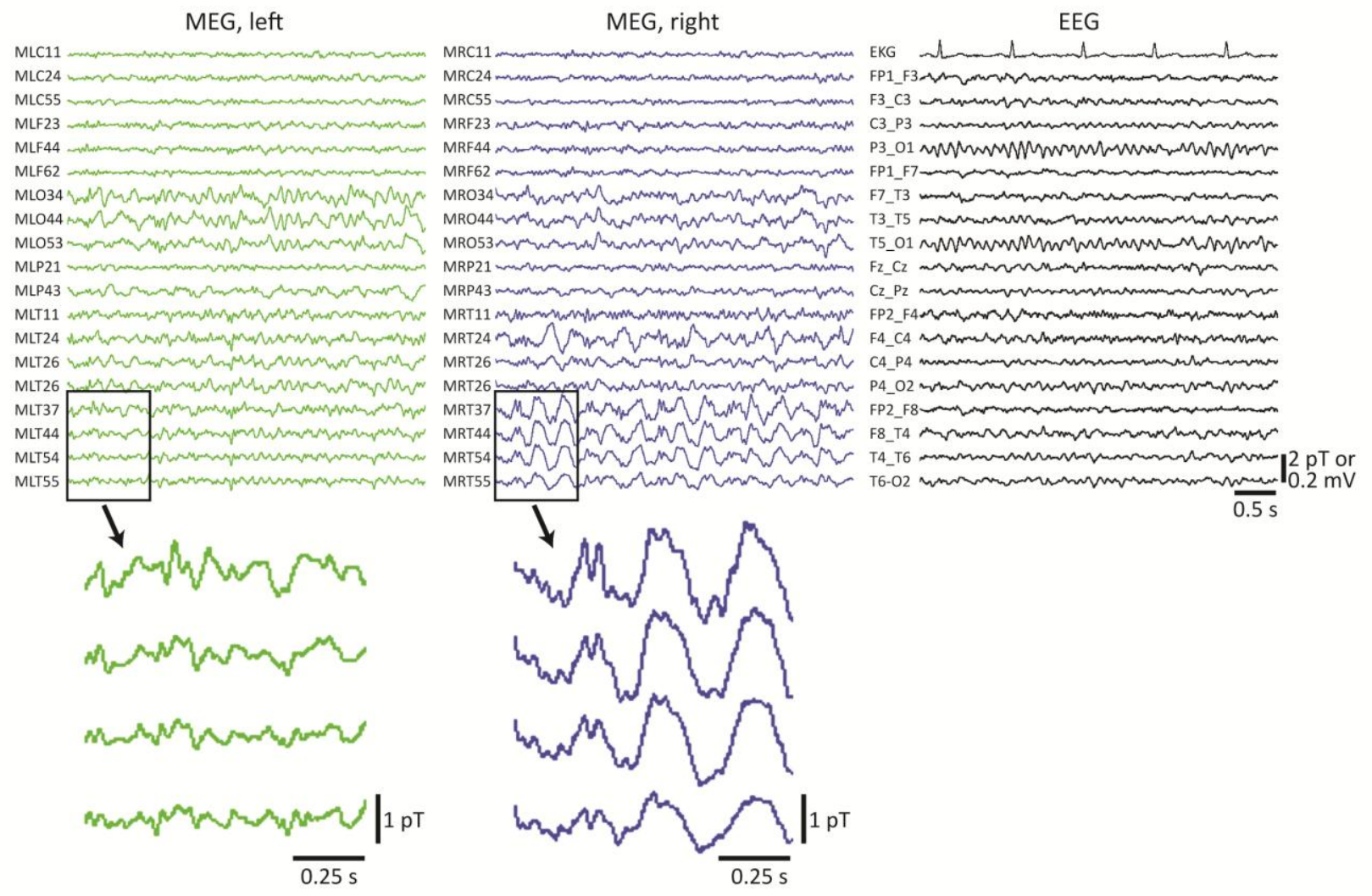

Figure 1 


\section{Asymmetric MEG Slowing EEG Slowing}

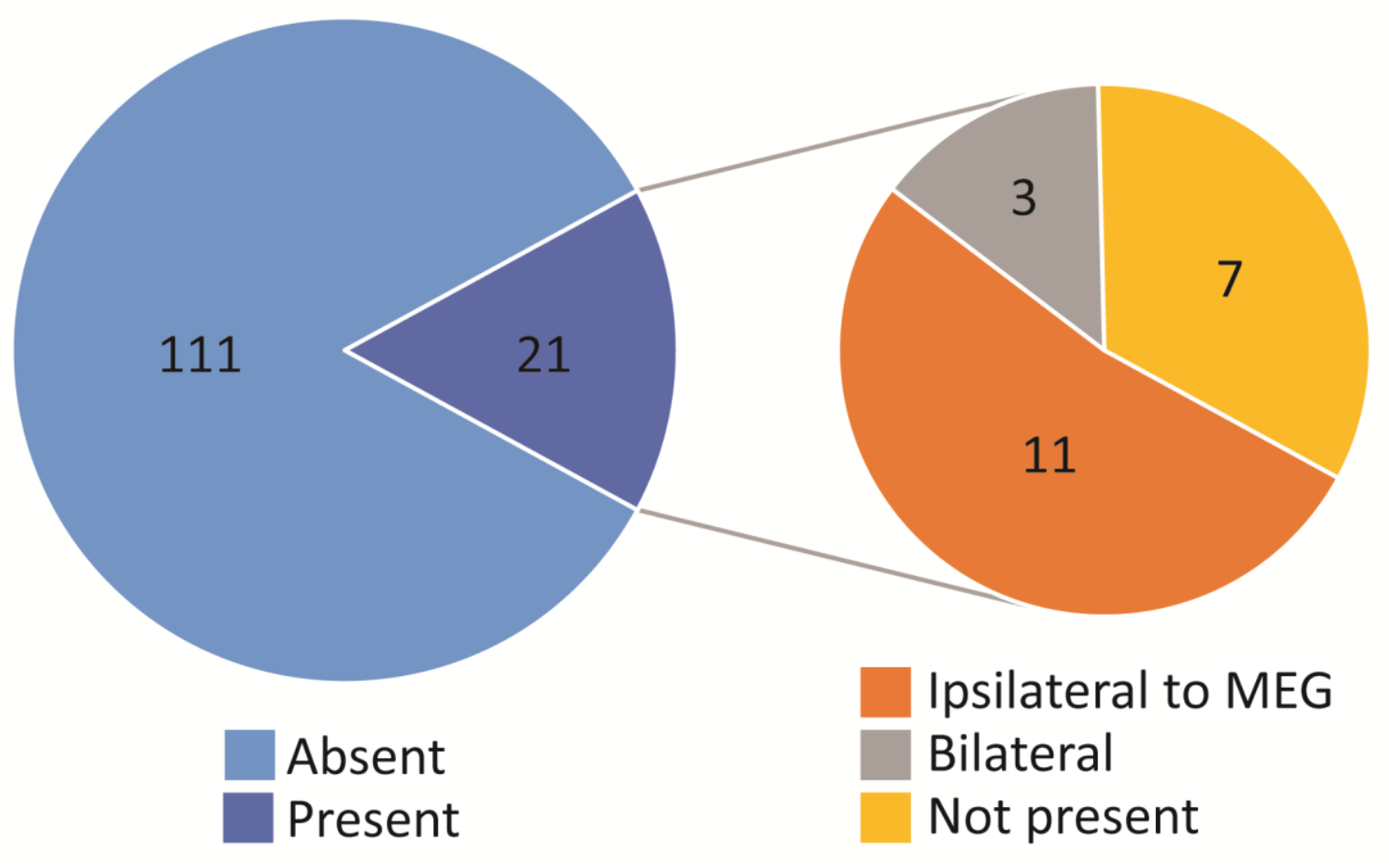

Figure 2 
Table 1: Characteristics of all patients

\begin{tabular}{llc}
\hline Age & years & $27.3 \pm 1.3$ \\
Gender & Male & $73(55.3)$ \\
& Female & $59(44.7)$ \\
Handedness & Right & $97(73.5)$ \\
& Left & $18(13.6)$ \\
Duration epilepsy & Ambidextrous/unknown & $17(12.9)$ \\
Seizure frequency & years & $14.0 \pm 1.0$ \\
Lobe involved & no. per week & $11.0 \pm 1.9$ \\
& Temporal & $75(56.8)$ \\
& Frontal & $27(20.5)$ \\
History of generalized seizures & Parietal & $7(5.3)$ \\
Side of surgery & Occipital & $5(3.8)$ \\
& Multiple & $18(13.6)$ \\
Previous resection & No & $78(59.1)$ \\
& Right & $54(40.9)$ \\
& Left & $55(41.7)$ \\
\hline
\end{tabular}

Data are $\mathrm{N}(\%)$ for categorical variables or mean \pm SEM for continuous variables. $\mathrm{N}=$ 132 patients. 
Table 2: Patients with asymmetric slowing on MEG

\begin{tabular}{|c|c|c|c|c|c|c|c|c|}
\hline $\begin{array}{l}\text { Patien } \\
\text { t no. }\end{array}$ & $\begin{array}{c}\mathbf{A g} \\
\mathrm{e}\end{array}$ & $\begin{array}{c}\text { Gende } \\
\mathbf{r}\end{array}$ & MEG slowing & $\begin{array}{c}\text { EEG } \\
\text { slowing }\end{array}$ & MEG spikes & $\begin{array}{c}\text { Resection } \\
\text { location }\end{array}$ & $\begin{array}{c}\text { Patholog } \\
\mathbf{y}\end{array}$ & $\begin{array}{c}\text { Enge } \\
1 \\
\text { class }\end{array}$ \\
\hline 1 & 3 & $\bar{F}$ & L hemisphere & bilateral & none seen & L occipital & $\mathrm{MCD}$ & 4 \\
\hline 2 & 3 & $\mathrm{~F}$ & $\begin{array}{c}\mathrm{L} \\
\text { frontotempora } \\
1\end{array}$ & L temporal & $\begin{array}{c}\mathrm{L} \\
\text { frontotemporal }\end{array}$ & L multilobe & gliosis & 1 \\
\hline 3 & 3 & M & L hemisphere & bilateral & $\mathrm{L}$ frontal & $\mathrm{L}$ frontal & gliosis & 3 \\
\hline 4 & 5 & M & $\mathrm{R}$ hemisphere & $\begin{array}{c}\mathrm{R} \\
\text { hemispher } \\
\mathrm{e}\end{array}$ & $\begin{array}{c}\mathrm{R} \\
\text { parietotempora } \\
1\end{array}$ & $\begin{array}{c}\mathrm{R} \\
\text { hemispherectom } \\
\mathrm{y}\end{array}$ & MCD & 1 \\
\hline 5 & 5 & M & $\mathrm{R}$ hemisphere & bilateral & $\mathrm{R}$ parietal & $\mathrm{R}$ parietal & MCD & 1 \\
\hline 6 & 6 & M & L hemisphere & $\begin{array}{c}\mathrm{L} \\
\text { hemispher } \\
\mathrm{e}\end{array}$ & $\begin{array}{c}\mathrm{L} \\
\text { frontotemporal }\end{array}$ & $\mathrm{L}$ frontotemporal & MCD & 1 \\
\hline 7 & 10 & M & $\mathrm{R}$ hemisphere & $\begin{array}{c}\mathrm{R} \\
\text { hemispher } \\
\mathrm{e}\end{array}$ & R occipital & R occipital & MCD & 1 \\
\hline 8 & 10 & M & L temporal & $\begin{array}{c}\mathrm{L} \\
\text { hemispher } \\
\mathrm{e}\end{array}$ & none seen & $\begin{array}{c}\mathrm{L} \\
\text { temporoparietal }\end{array}$ & MCD & 1 \\
\hline 9 & 10 & M & L hemisphere & $\begin{array}{c}\mathrm{L} \\
\text { hemispher } \\
\mathrm{e}\end{array}$ & $\mathrm{L}$ frontal & $\mathrm{L}$ frontal & MCD & 1 \\
\hline 10 & 10 & M & $\begin{array}{c}\mathrm{L} \\
\text { frontotempora } \\
1\end{array}$ & L temporal & L temporal & L temporal & MTS & 1 \\
\hline 11 & 11 & $\mathrm{~F}$ & R temporal & $\mathrm{R}$ temporal & $\mathrm{R}$ temporal & $\mathrm{R}$ temporal & MTS & 1 \\
\hline 12 & 13 & $\mathrm{~F}$ & $\mathrm{R}$ hemisphere & $\begin{array}{c}\mathrm{R} \\
\text { hemispher } \\
\mathrm{e}\end{array}$ & $\mathrm{R}$ frontal & $\mathrm{R}$ frontal & MCD & 1 \\
\hline 13 & 15 & M & $\mathrm{R}$ parietal & none seen & $\mathrm{R}$ parietal & $\mathrm{R}$ parietal & gliosis & 2 \\
\hline 14 & 16 & M & L hemisphere & $\begin{array}{c}\mathrm{L} \\
\text { hemispher } \\
\mathrm{e}\end{array}$ & $\mathrm{L}$ insula & L insula & MCD & 3 \\
\hline
\end{tabular}




\begin{tabular}{|c|c|c|c|c|c|c|c|c|}
\hline \multirow[t]{2}{*}{15} & & M & L temporal & $\begin{array}{c}\text { L } \\
\text { hemispher }\end{array}$ & none seen & \multicolumn{3}{|c|}{ gliosis } \\
\hline & 26 & & & $\mathrm{e}$ & & L temporal & & 3 \\
\hline 16 & 26 & M & $\mathrm{R}$ hemisphere & none seen & $\begin{array}{c}\mathrm{L} \\
\text { parietotempora } \\
1\end{array}$ & L temporal & MTS & 1 \\
\hline 17 & 30 & M & L temporal & none seen & $\begin{array}{c}\mathrm{L} \\
\text { frontotemporal }\end{array}$ & L temporal & MTS & 1 \\
\hline $18^{*}$ & 33 & M & $\mathrm{R}$ temporal & none seen & none seen & $\mathrm{R}$ temporal & gliosis & 1 \\
\hline 19 & 43 & $\mathrm{~F}$ & $\mathrm{R}$ temporal & none seen & R temporal & $\mathrm{R}$ temporal & MCD & 3 \\
\hline 20 & 49 & $\mathrm{~F}$ & L temporal & $\begin{array}{c}\text { none } \\
\text { seen** }\end{array}$ & L temporal & L insula & tumor & 1 \\
\hline 21 & 51 & $\mathrm{~F}$ & $\mathrm{R}$ hemisphere & none seen & $\mathrm{R}$ parietal & $\mathrm{R}$ parietal & ischemia & 1 \\
\hline
\end{tabular}

*Patient shown in Figure 1. **Based on simultaneous MEG/EEG only; long-term EEG not done. F: female; L: left; M: male; MCD: malformation of cortical development; MTS: mesial temporal sclerosis; R: right. 
Table 3: Seizure outcomes and associated factors

\begin{tabular}{|c|c|c|c|c|}
\hline & & $\begin{array}{c}\text { MEG } \\
\text { slowing }\end{array}$ & $\begin{array}{c}\text { No MEG } \\
\text { slowing }\end{array}$ & $p$ value \\
\hline \multicolumn{5}{|l|}{ A) Patient demographics } \\
\hline Age & years & $20.0 \pm 3.3$ & $28.2 \pm 1.4$ & $<0.01 *$ \\
\hline$\underline{\text { Gender }}$ & $\begin{array}{l}\text { Male } \\
\text { Female }\end{array}$ & $\begin{array}{l}14(19.2) \\
7(11.9)\end{array}$ & $\begin{array}{l}59(80.8) \\
52(88.1)\end{array}$ & 0.34 \\
\hline$\underline{\text { Handedness }}$ & $\begin{array}{l}\text { Right } \\
\text { Left } \\
\text { Ambidextrous/unknown }\end{array}$ & $\begin{array}{l}13(13.4) \\
2(11.1) \\
6(35.3)\end{array}$ & $\begin{array}{l}84(86.6) \\
16(88.9) \\
11(64.7)\end{array}$ & 0.06 \\
\hline \multicolumn{5}{|l|}{ B) Epilepsy characteristics } \\
\hline Duration epilepsy & years & $8.0 \pm 1.5$ & $15.2 \pm 1.1$ & $<0.01 *$ \\
\hline Seizure frequency & no. per week & $13.1 \pm 3.8$ & $10.7 \pm 2.2$ & 0.65 \\
\hline AEDs tried & no. & $5.7 \pm 0.5$ & $4.7 \pm 0.3$ & 0.13 \\
\hline Lobe involved & $\begin{array}{l}\text { Temporal } \\
\text { Extratemporal/multilobe }\end{array}$ & $\begin{array}{c}7(9.3) \\
14(24.6)\end{array}$ & $\begin{array}{l}68(90.7) \\
43(75.4)\end{array}$ & $0.03^{*}$ \\
\hline Primary Pathology & $\begin{array}{l}\text { Gliosis only } \\
\text { MCD } \\
\text { MTS }\end{array}$ & $\begin{array}{c}5(11.9) \\
10(30.3) \\
4(16.0)\end{array}$ & $\begin{array}{l}37(88.1) \\
23(69.7) \\
21(84.0)\end{array}$ & 0.12 \\
\hline & $\begin{array}{l}\text { Tumor } \\
\text { Ischemia/infarct } \\
\text { Other }\end{array}$ & $\begin{array}{l}1(4.5) \\
1(20.0) \\
0(0.0)\end{array}$ & $\begin{array}{l}21(95.5) \\
4(80.0) \\
5(100.0)\end{array}$ & \\
\hline History of generalized seizures & $\begin{array}{l}\text { Yes } \\
\text { No }\end{array}$ & $\begin{array}{l}13(16.7) \\
8(14.8)\end{array}$ & $\begin{array}{l}65(83.3) \\
46(85.2)\end{array}$ & 0.81 \\
\hline Side of surgery & $\begin{array}{l}\text { Right } \\
\text { Left }\end{array}$ & $\begin{array}{l}9(26.4) \\
12(15.6)\end{array}$ & $\begin{array}{l}46(83.6) \\
65(84.4)\end{array}$ & 0.99 \\
\hline$\underline{\text { Previous resection }}$ & $\begin{array}{l}\text { No } \\
\text { Yes }\end{array}$ & $\begin{array}{l}18(16.1) \\
3(15.0)\end{array}$ & $\begin{array}{l}94(83.9) \\
17(85.0)\end{array}$ & 0.99 \\
\hline \multicolumn{5}{|l|}{ C) Pre-operative diagnostics } \\
\hline MEG spike activity & $\begin{array}{l}\text { Specific to EZ } \\
\text { Non-specific or discordant } \\
\text { No spikes modelled }\end{array}$ & $\begin{array}{l}12(17.6) \\
5(14.3) \\
4(13.8)\end{array}$ & $\begin{array}{l}56(82.4) \\
30(85.7) \\
25(86.2)\end{array}$ & 0.85 \\
\hline$\underline{\text { MRI }}$ & $\begin{array}{l}\text { Abnormal } \\
\text { Normal }\end{array}$ & $\begin{array}{l}18(16.5) \\
3(13.0)\end{array}$ & $\begin{array}{l}91(83.5) \\
20(87.0)\end{array}$ & 0.48 \\
\hline Ictal scalp EEG & $\begin{array}{l}\text { Localized } \\
\text { Not localized }\end{array}$ & $\begin{array}{l}13(16.5) \\
8(16.0)\end{array}$ & $\begin{array}{l}66(83.5) \\
42(84.0)\end{array}$ & 0.99 \\
\hline PET (when performed) & $\begin{array}{l}\text { Abnormal } \\
\text { Normal }\end{array}$ & $\begin{array}{l}4(15.4) \\
2(15.4)\end{array}$ & $\begin{array}{l}22(84.6) \\
11(84.6)\end{array}$ & 0.99 \\
\hline TOTAL & & $21(15.9)$ & $111(84.1)$ & \\
\hline
\end{tabular}

Data are $\mathrm{N}(\%)$ for categorical variables or mean \pm SEM for continuous variables. *Statistically significant value $(p$ $<0.05$ ) from $\chi^{2}$ test (categorical) or $t$-test (continuous) comparing patients with or without asymmetric slowing on MEG. AED: anti-epileptic drug; EEG: electroencephalography; EZ: epileptogenic zone; MCD: malformation of cortical development; MEG: magnetoencephalography; MRI: magnetic resonance imaging; MTS: mesial temporal sclerosis; PET: positron emission tomography. 